\title{
Spike Sorting with Support Vector Machines
}

\author{
R. Jacob Vogelstein*, Kartikeya Murari*, Pramodsingh H. Thakur*, \\ Gert Cauwenberghs $^{\dagger}$, Shantanu Chakrabartty ${ }^{\dagger}$, and Chris Diehl ${ }^{\dagger \ddagger}$ \\ *Department of Biomedical Engineering \\ ${ }^{\dagger}$ Department of Electrical and Computer Engineering \\ ${ }_{\ddagger}$ Applied Physics Laboratory \\ Johns Hopkins University, Baltimore, MD 21218 \\ \{jvogelst,kartik,pramod,gert,shantanu\}@jhu.edu, chris.diehl@jhuapl.edu
}

\begin{abstract}
Spike sorting of neural data from single electrode recordings is a hard problem in machine learning that relies on significant input by human experts. We approach the task of learning to detect and classify spike waveforms in additive noise using two stages of large margin kernel classification and probability regression. Controlled numerical experiments using spike and noise data extracted from neural recordings indicate significant improvements in detection and classification accuracy over amplitude- and linear template-based spike sorting techniques.
\end{abstract}

\section{INTRODUCTION}

Recording electrical activity from neurons in the brain has become an indispensable technique in modern neuroscience research. Typically, these recordings are obtained by advancing a metal probe through neural tissue until a neuron of interest is located. It is difficult to position an electrode in such a way as to isolate a single neuron, so the activity recorded is frequently derived from multiple neural sources. Unless the contribution of each source can be separated from the others (and from background noise), the integrity of the experiment may be compromised. A number of efforts (reviewed in [1], [2]) have been directed at this problem of neural source separation - commonly called "spike sorting" — but none has been completely effective in all situations. In fact, although new approaches look promising [3]-[6], some of the simplest measures have been most successful [7].

Spike sorting systems rely on the fact that the waveforms ("spikes") recorded from a specific neuron are functions of both the intrinsic electrochemical dynamics of that neuron and the position of the electrode with respect to the neuron. Furthermore, they assume that in a noiseless system, each recorded spike from a given neuron over a short period of time would be nearly identical, although spikes from different neurons could vary in shape. In reality, though, the system has many sources of noise — slight perturbations in electrode position, activity of distant cells, environmental factors, etc. - and consequently every recorded spike appears different. Moreover, because the spectral contents of spikes and noise are similar, many recorded spikes appear similar to noise, and vice-versa. The ability to distinguish spikes from noise ("spike detection"), and to distinguish spikes from different sources ("spike classification"), therefore depends on both the disparities between the noise-free spikes from each source ("templates") and the signal-to-noise level (SNR) in the recording system. An additional factor that we will not consider as a variable in this paper (but see [3], [8]) is the overall activity level of the neurons, which affects the number of coincident ("overlapping") spikes.

In the following sections we describe a novel spike sorting architecture based on GiniSVM support vector machine classification and probability regression [9]. The spike sorter is evaluated with numerical experiments on spike and noise data extracted from neural recordings over a large range of signalto-noise ratios and template disparities, and demonstrates superior performance to standard template matching techniques.

\section{MethodS}

\section{A. Experimental Methods}

Electrophysiology recordings were made in male and female rhesus monkeys (Macaca mulatta), each weighing 4-5 kg, during a sensory neurophysiology experiment. On each recording day, single and multiple units were isolated in cortical areas 1 and $3 b$ using quartz-coated platinum/tungsten (90/10) electrodes (diameter, $80 \mathrm{~mm}$; tip diameter, $4 \mathrm{~mm}$; and impedance, $15 \mathrm{M} \Omega$ at $1000 \mathrm{~Hz}$ ). The raw data were filtered and amplified before being digitized (National Instruments PCI-6052) at a $40 \mathrm{KHz}$ sampling rate and stored. All surgical procedures were done under sterile conditions and in accordance with the rules and regulations of the Johns Hopkins Animal Care and Use Committee and the Society for Neuroscience.

During off-line analysis of each recording session, pure noise segments and segments containing putative spikes were automatically identified and manually verified [10], [11]. Putative spike waveforms were time-aligned and clustered in a principal component space under human supervision; templates were formed by averaging all points sufficiently close together. Out of 81 recording sessions, 25 were found to contain two or more templates.

\section{B. Data Generation}

A persistent issue in the spike sorting literature is whether to use real or simulated data to test new algorithms. While using real data would be preferable in some ways, real data is fundamentally unlabeled, so it necessitates testing the algorithm using possibly-incorrect labels supplied by a human expert. Therefore, simulated data is used in many 
Dataset 16

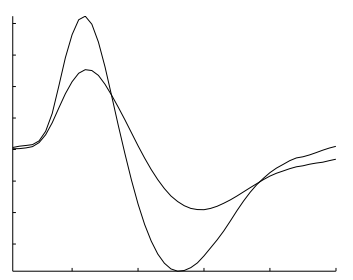

Dataset 37
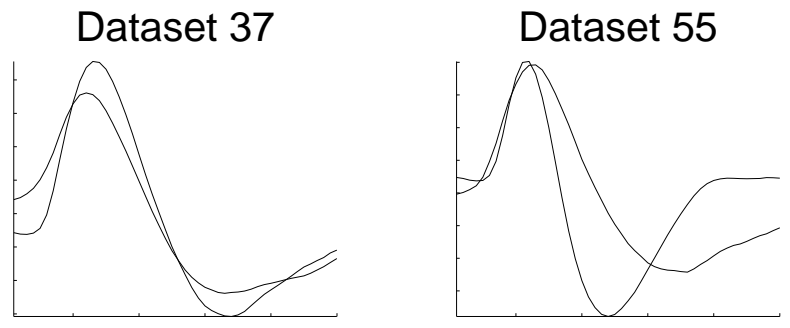

(a)
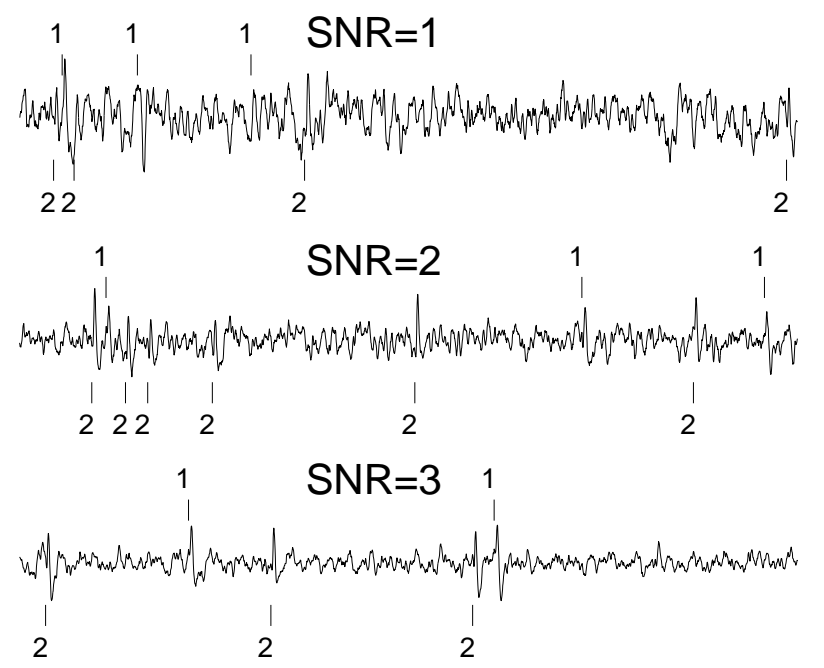

(b)

Fig. 1. (a) The four sets of experimentally-recorded templates used in the generative model for all data in this paper. (b) Typical simulated recordings at $\mathrm{SNR}=1$ (top), $\mathrm{SNR}=2$ (middle), and $\mathrm{SNR}=3$ (bottom). Spike locations are labeled with the neuron class.

spike-sorting publications (e.g. [3], [4], [8], [12]). The lack of standards and publicly-available databases with labeled spikes complicate comparisons between different methods in the published literature. We have created a generative model to simulate a neural recording based on parameters measured from actual recordings, and will gladly provide our simulated data upon request.

1) Spikes and Noise: We simulate a neural recording by inserting real spike templates into a background process of stationary colored Gaussian noise. The background noise process can be completely described by its standard deviation and noise autocorrelation vector. To increase the accuracy of the model, we estimate both of these parameters from pure noise segments of real neural recordings (see Section II-A) and then simulate the noise using an autoregressive filter [13]. Spline- interpolated spike templates (with random phase) are inserted according to a modified Poisson process where the number of spikes in a fixed time period is given by the usual Poisson probability distribution, but the inter-spike interval is not a true exponential random variable because of the refractory period of the neurons. For all of the experiments described below, each simulated recording uses a randomly selected set of noise parameters taken from a real recording session. In order to draw fair comparisons across multiple signal-to-noise ratios, we have selected four representative sets of templates, shown in Figure 1a.

After selecting a set of noise statistics and templates for a simulation, we also specify the signal-to-noise ratio (SNR). Although there are many ways of calculating this value, we define it as the root mean squared value of the template divided by the standard deviation of the simulated noise, i.e. SNR = $\|$ template $\| / \sigma \cdot \sqrt{\mid \text { template } \mid}$ where $\|\cdot\|$ is the $\mathrm{L}_{2}$ norm, $\sigma$ is the standard deviation of the simulated noise, and $|\cdot|$ is the length in number of samples.

\section{Detection and Classification}

The spike sorting system consists of two stages - detection and classification - each trained using a support vector machine (SVM) classifier. The first stage discriminates between noise and the occurrence of a spike over time, and the second stage discriminates between spike templates.

GiniSVM [14], a sparse large-margin kernel machine for logistic probability regression, is used to estimate class output probabilities. The class probabilities yield confidence values for the classified spike outputs, and are used in expectation-maximization based training of partially-labeled data. The quadratic form of entropy in the dual formulation of GiniSVM offers sparsity in the kernel representation, and corresponds to a Huber loss function in the primal formulation [9]. Class probabilities in the binary case take the form

$$
P(1 \mid \mathbf{x})=\frac{1}{1+e^{-(\mathbf{w} \Phi(\mathbf{x})+b)}}=\frac{1}{1+e^{-\left(\sum_{i} y_{i} \alpha_{i} k\left(\mathbf{x}, \mathbf{x}_{i}\right)+b\right)}}
$$

and $P(-1 \mid \mathbf{x})=1-P(1 \mid \mathbf{x})$, where $x$ is the vector to be classified, $x_{i}$ are training vectors, $y_{i}= \pm 1$ are the corresponding class labels, and $k(\cdot, \cdot)$ defines the kernel. Binary GiniSVM minimizes the following objective function in the dual coefficients $\alpha_{i}$ :

$$
\begin{array}{r}
\min _{\alpha}: \frac{1}{2} \sum_{i, j} \alpha_{i}\left(Q_{i j}+\frac{8 \gamma}{C_{i}} \delta_{i j}\right) \alpha_{j}-4 \gamma \sum_{i} \alpha_{i} \\
\text { subject to } \sum_{i} y_{i} \alpha_{i}=0 \text { and } 0 \leq \alpha_{i} \leq C_{i}, \forall i
\end{array}
$$

where $Q_{i j}=y_{i} y_{j} k\left(\mathbf{x}_{i}, \mathbf{x}_{j}\right)$ is the kernel matrix evaluated at training vectors $i$ and $j, \gamma$ defines the margin, $C_{i}$ are (data-dependent) regularization constants, and $\delta_{i j}=1$ for $i=j$ and zero otherwise. GiniSVM offers the additional computational advantage that it is compatible with standard quadratic programming techniques for SVM training. 
For comparison purposes, we also perform spike detection by simple amplitude thresholding and spike classification using a standard template matching technique [2]. For template matching, we average all spikes observed from a given neuron in the SVM training set and use the mean waveforms as the templates. Decisions are based on the Euclidian, Mahalanobis, or PCA distances between an input vector and the spike templates, i.e. an input vector is assigned the label of whichever template is closest.

\section{Performance Measures}

To test the performance of the detection stage, we analyze 10 seconds of simulated stationary neural recordings captured immediately after the initial two seconds used for training the system. A sliding window of $1.25 \mathrm{msec}$ duration is moved across the data and each epoch is evaluated by the SVM. The resulting output is a sequence of probabilities of the given epochs being "spikes" versus "noise". The performance metric sets a threshold at five times the standard deviation of this signal and calculates merit as the ratio of the difference between hits and false positives to the total number of spikes, where a "hit" is assessed whenever the mean time between successive SVM output threshold crossings occurs within $\pm 0.2 \mathrm{msec}$ of an actual spike time, and a "false positive" is assessed for all mean threshold crossing times outside this range.

To test classification performance, we evaluate the fraction of correctly classified spikes in the second stage, assuming correct detection in the first stage. Since the exact spike times are known for the simulated data, we extract $1.25 \mathrm{msec}$ of data beginning from each spike onset and use these as the input to the SVM and template matching algorithms.

\section{RESULTS}

1) Detection Stage: For the detection stage, training data is generated from the first two seconds of a simulated neural recording, where each training vector consists of $1.25 \mathrm{msec}$ (50 samples at a $40 \mathrm{KHz}$ sampling rate). Training data include centered spikes as "spike" vectors, surround of spikes as "noise" vectors, and pure noise; 500 examples from each class are used, for a total of 1,500 training vectors. Results from the detection stage are shown in Figure 2a, which plots "merit" (see Section II-D) against signal-to-noise ratio (SNR). Each point on these lines represents the average performance on ten seconds of simulated data, where averages are taken across all four sets of templates (Figure 1a). For a SNR $=2$, the SVM detection stage provides better than $80 \%$ accuracy compared to less than $60 \%$ for amplitude thresholding, and by SNR $=3$ it has reached its asymptotic performance level of about $95 \%$. In comparison, thresholding does not reach an equivalent level until a $\mathrm{SNR}=5$. The middle axis of Figure $1 \mathrm{~b}$ shows a recording with SNR $=2-$ at this level, the decisions are not obvious to the untrained eye, but the SVM is very effective. For a SNR between zero and one, both basic amplitude thresholding and SVM detector perform

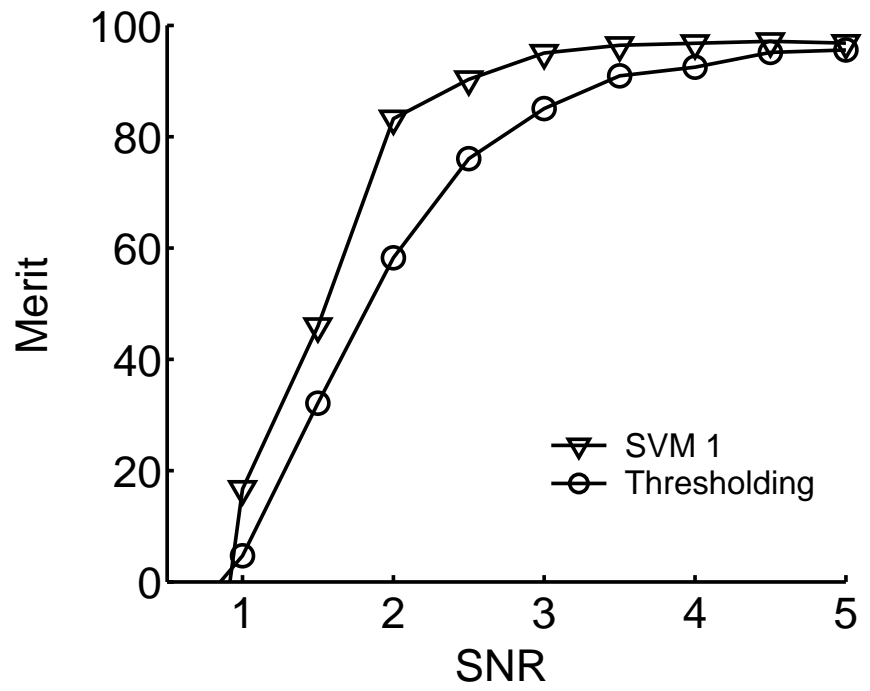

(a)

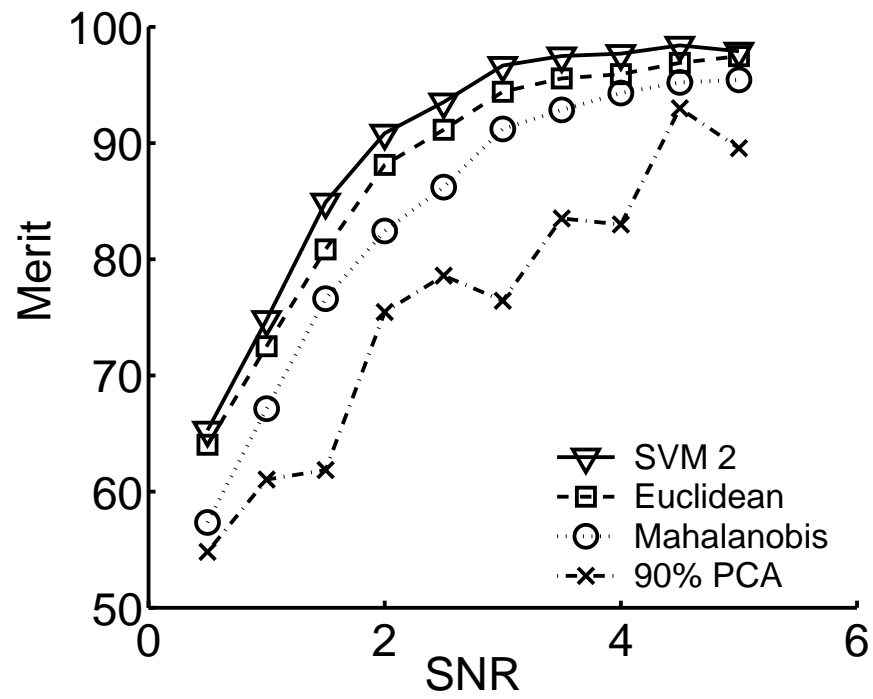

(b)

Fig. 2. Performance as a function of SNR for (a) detection stage and (b) classifi cation stage. The "90\% PCA" curve illustrates the results if template distance is calculated in a lower-dimensional space where dimensions are chosen to account for approximately $90 \%$ of the variance of the data, as given by standard principal component analysis techniques.

poorly, with a greater number of false positives than correctly classified spike epochs.

2) Classification Stage: The results of the classification stage, trained over the initial five seconds of data and assuming perfect detection, are summarized in Figure 2b. The SVM classifier consistently outperforms template matching over the entire range of SNRs tested, although it only exceeds the Euclidean distance metric by a slight margin. Both techniques appear to reach an asymptotic success rate of about $95 \%$. This seems reasonable, as no precautions are taken to avoid simulating overlapping spikes, and destructive interference is likely to render decision making impossible occasionally. Figure $1 \mathrm{~b}$ provides an example of some typical test data, and 


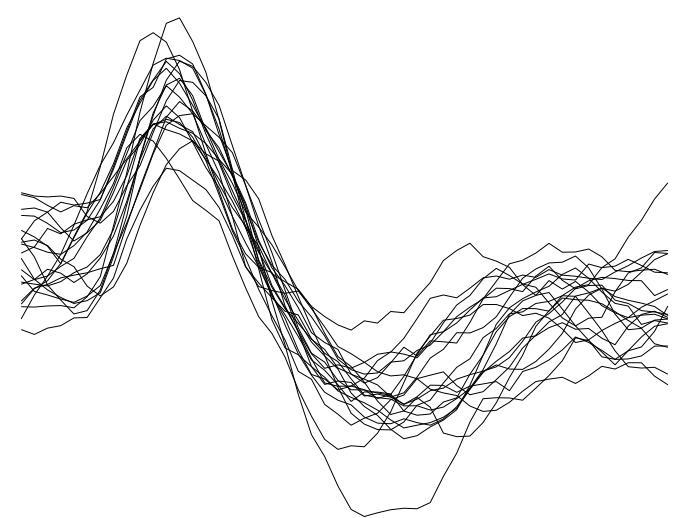

(a)

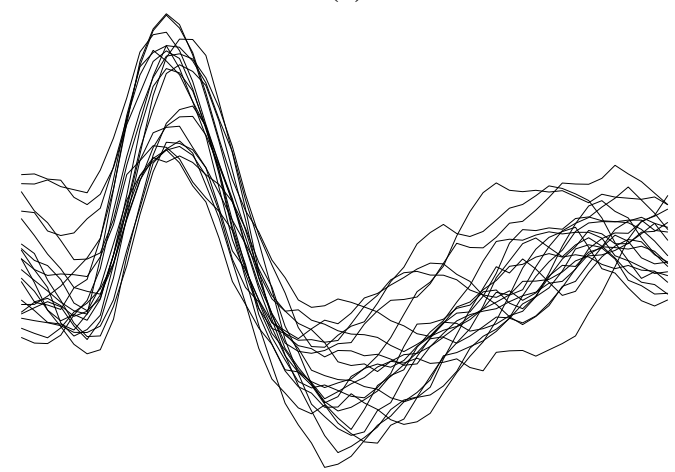

(b)

Fig. 3. Sample data from a SNR $=2$ dataset determined by the $\mathrm{SVM}$ to be (a) class 1 and (b) class 2 . Out of the 40 spikes shown here, 3 are misclassifi ed.

Figure 3 illustrates some of the decisions made by the classifier on a $\mathrm{SNR}=2$ dataset.

\section{CONCLUSION}

We have demonstrated the success of a novel approach to neural spike sorting using support vector machines. For our simulated data, the SVM classifiers outperform standard methods in both the detection and classification stages. Future work will focus on using an EM-based transductive form of SVM training to deal with nonstationary and partially-labeled data.

\section{ACKNOWLEDGMENT}

The authors would like to thank Ken Johnson for supervising the work of PHT on the generative model and for his insightful comments regarding this work.

\section{REFERENCES}

[1] M. S. Lewicki, "A review of methods for spike sorting: the detection and classifi cation of neural action potentials," Network, vol. 9, pp. R53-R78, 1998.

[2] B. C. Wheeler, "Automatic discrimination of single units," in Methods for neural ensemble recordings, M. Nicolelis, Ed. Boca Raton, Florida: CRC Press, 1999, ch. 4, pp. 61-78.

[3] R. Chandra and L. M. Optican, 'Detection, classifi cation, and superposition resolution of action potentials in multiunit single-channel recordings by an on-line real-time neural network," IEEE Trans. on Biomed. Engin., vol. 44, no. 5, pp. 403-412, 1997.

[4] K. G. Oweiss and D. J. Anderson, 'Spike sorting: a novel shift and amplitude invariant technique," Neurocomputing, vol. 44-46, pp. 1133$1139,2002$.

[5] K. H. Kim and S. J. Kim, 'Neural spike sorting under nearly 0-db signal-to-noise ratio using nonlinear energy operator and artifi cial neuralnetwork classifi er," IEEE Trans. on Biomed. Engin., vol. 47, no. 10, pp. $1406-1411,2000$

[6] E. Hulata, R. Segev, and E. Ben-Jacob, "A method for spike sorting and detection based on wavelet packets and shannon's mutual information," J. Neurosci. Methods, vol. 117, no. 1, pp. 1-12, 2002.

[7] B. C. Wheeler and W. J. Heetderks, "A comparison of techniques for classifi cation of multiple neural signals," IEEE Trans. Biomed. Engin., vol. 29, pp. 752-759, 1987.

[8] M. S. Lewicki, 'Bayesian modeling and classifi cation of neural signals,' Neural Comput., vol. 6, no. 5, pp. 1005-1030, 1994.

[9] S. Chakrabartty and G. Cauwenberghs, "Forward decoding kernel machines: A hybrid hmm/svm approach to sequence recognition," in Proc. SVM 2002, S.-W. Lee and A. Verri, Eds. Springer, 2002, pp. 278-292.

[10] H. Lu, 'Neural spike detection and classifi cation," Master's thesis, Johns Hopkins University, 1999.

[11] P. H. Thakur, "automated optimal detection and classifi cation of neural action potentials in extra-cellular recordings," Master's thesis, Johns Hopkins University, 2002.

[12] K. M. L. Menne, A. Folkers, T. Malina, R. Maex, and U. G. Hoffman, "Test of spike-sorting algorithms on the basis of simulated network data," Neurocomputing, vol. 44-46, pp. 1119-1126, 2002.

[13] G. E. P. Box and G. M. Jenkins, Time series analysis: forecasting and control, 1st ed. Oakland: Holden-Day, 1976.

[14] S. Chakrabartty and G. Cauwenberghs, 'Forward-decoding kernel-based phone sequence recognition," in Advances in Neural Information Processing Systems, S. Becker, S. Thrus, and K. Obermayer, Eds., vol. 15. Cambridge, MA: MIT Press, 2003. 